\title{
Commentary Expanding Our Understanding of Neurobiological Mechanisms of Resilience by Using Animal Models
}

\author{
Israel Liberzon*,',2 and Dayan Knox' \\ 'Department of Psychiatry, University of Michigan, Ann Arbor, MI, USA; ${ }^{2}$ Veterans Affairs Hospital, Ann Arbor, MI, USA
}

Neuropsychopharmacology (2012) 37, 317-318; doi:10.1038/npp.2011.259

The original conceptualization of stress and the stress response has been developed based on the understanding that certain environmental exposures and life events can be both detrimental to individual's health and wellbeing, and also promote physiological responses that can be adaptive. Interestingly, until recently, the majority of translational research focused on neurobiological processes involved in the abnormal or altered stress response, on the vulnerability to stress, or the negative consequences of stress. Only recently the field started focusing on the concept of resilience, exploring the possibility that, similarly to stress vulnerability, there could be unique mechanisms involved in resilience to stress. Defining resilience has not been easy and within clinical research, resilience often refers to the ability of an organism to deal with, or adapt to, the pathological effects of stress. This seemingly subtle shift in research focus actually has immediate and substantial implications to stress research, as it suggests that some individuals are particularly adept to dealing with stressful situations, and if we examine this subgroup of individuals, we are likely to find different or compensatory changes, or changes in biological processes that are diametrically opposed to those found in stress-vulnerable individuals.

It has long been known that significant individual variability in vulnerability to severe stress or trauma exists with respect to the development of psychiatric consequences like post-traumatic stress disorder (PTSD), however, the resilience perspective might suggest that this can be accounted for by individual variability in resilience. Insofar as the mechanisms of vulnerability and resilience do not overlap completely, the study of the neurobiology of resilience is not simply a mirror of the study of stress vulnerability, but it could offer new perspectives on pathophysiology or treatment of stress-induced anxiety disorders such as PTSD and depression. Together with the study of stress vulnerability, understanding the neurobiology of resilience could

*Correspondence: Dr I Liberzon, Department of Psychiatry, University of Michigan, Rachel Upjohn Building, 4250 Plymouth Rd, Ann Arbor, MI 48I09-5765, USA, Tel: + I 734764 9527, Fax: + I 7349367868 , E-mail: Liberzon@med.umich.edu

Received 19 September 201 I; accepted 19 September 2011 provide a more comprehensive picture in the service of understanding pathophysiology, development of new treatments for stress-induced psychiatric disorders, and identifying markers for primary and secondary prevention. More importantly, if individuals who have low levels of resilience can be treated in such a manner so as to increase their levels of resilience, this could greatly reduce the incidence of stress-induced psychiatric disorders.

Understanding the neurobiology of resilience in clinical research can be hindered by ethical constraints, but can be readily explored by employing animal models of stress and trauma. One promising approach for studying the neurobiology of resilience is the use of the cut-off behavioral criteria $(\mathrm{CBC})$, as proposed by Cohen et al. This method, here applied to predator-odor-exposed animals that are later tested for startle and anxiety behavior, allows for identification of not only vulnerable groups (extreme behavioral response) but also resilient subgroups (minimal behavioral response). By examining experimental procedures that increase the incidence of minimal responders after predator odor exposure, neurobiological mechanisms of resilience can be explored.

In a recent study by Cohen et al. (2011), the authors used predator odor exposure with $\mathrm{CBC}$ to demonstrate that neuropeptide Y (NPY) expression in hippocampus, periaqueductal gray, and amygdala are associated with resilience to predator odor effects. The authors also report that NPY infusion into the hippocampus, $1 \mathrm{~h}$ following predator odor exposure, leads to a larger ratio of resilient animals whereas blockade of the NPY-1 receptor in the hippocampus decreases the incidence of resilient behavior. These findings suggest that NPY-1 receptor activation in the hippocampus is involved in, or even critical for, the development of resilience after stress exposure. These results are in agreement with previous reports of association of NPY plasma levels and resilience to traumatic stress in human subjects (Yehuda et al, 2006) and polymorphisms in the NPY gene and resilience in non-human primates (Lindell et al, 2010). Where the study by Cohen et al. (2011) adds to the literature about NPY and resilience is the specific mechanism and the neuroanatomical region (ie, enhanced activation of the NPY1 receptor in the 
hippocampus) for NPY effects. The hippocampus is critical for contextual processing, and we had argued that contextual processing is central in PTSD (Liberzon and Sripada, 2008). There is also a well-documented role for the hippocampus in stress responsivity, and although significant controversy regarding hippocampal volume abnormalities in PTSD existed in the literature, accumulating evidence confirms hippocampal abnormalities in PTSD. Various studies of animal models of PTSD have consistently implicated hippocampal abnormalities in PTSD, and the changes in brain-derived neurotropic factor (BDNF) and its receptor (TrkB) signaling in the hippocampus have been reported both in SPS (Takei et al, 2011) and predatorexposure models (Roth et al, 2011). Cohen et al. (2011) further extended these findings by reporting increases in BDNF and a decrease in glucocorticoid receptor expression in the hippocampus with increased NPY expression. The link between NPY and glucocorticoid signaling has been reported in SPS animal model of PTSD (Cui et al, 2008), however, additional research is needed to establish the link between NPY-1 receptor activation in the hippocampus and changes in BDNF and glucocorticoid receptor expression.

The animal models of PTSD studies have been helpful in identifying target mechanisms involved in PTSD pathophysiology and possible vulnerability. The role of glucocorticoid, serotonergic, glutamatergic, and catecholaminergic systems have been consistently implicated in vulnerability and resilience to stress, and is continuously studied. Application of $\mathrm{CBC}$ by Cohen et al. brings a new dimension to this effort by creating an opportunity to focus on highly resilient groups, and highlighting novel neurobiological targets like NPY in the hippocampus for the study of neurobiology of resilience.

\section{DISCLOSURE}

The author declares no conflict interest.

\section{REFERENCES}

Cohen H, Tianmin L, Kozlovsky N, Zeev K, Joseph Z, Aleksander MA (2011). The neuropeptide Y(NPY)-ergic system is associated with behavioral resilience to stress exposure in an animal model of posttraumatic stress disorder. Neuropsychopharmacology 37: 350-363.

Cui H, Sakamoto H, Higashi S, Kawata M (2008). Effects of singleprolonged stress on neurons and their afferent inputs in the amygdala. Neuroscience 152: 703-712.

Liberzon I, Sripada CS (2008). The functional neuroanatomy of PTSD: a critical review. Prog Brain Res 167: 151-169.

Lindell SG, Schwandt ML, Sun H, Sparenborg JD, Bjork K, Kasckow JW et al. (2010). Functional NPY variation as a factor in stress resilience and alcohol consumption in rhesus macaques. Arch Gen Psychiatry 67: 423-431.

Roth TL, Zoladz PR, Sweatt JD, Diamond DM (2011). Epigenetic modification of hippocampal Bdnf DNA in adult rats in an animal model of post-traumatic stress disorder. J Psychiatr Res 45: 919-926.

Takei S, Morinobu S, Yamamoto S, Fuchikami M, Matsumoto T, Yamawaki S (2011). Enhanced hippocampal BDNF/TrkB signaling in response to fear conditioning in an animal model of posttraumatic stress disorder. J Psychiatr Res 45: 460-468.

Yehuda R, Brand S, Yang RK (2006). Plasma neuropeptide Y concentrations in combat exposed veterans: relationship to trauma exposure, recovery from PTSD, and coping. Biol Psychiatry 59: 660-663. 